\title{
Extraction of tidal channel networks from airborne scanning laser altimetry and aerial photography
}

Book or Report Section

Accepted Version

Mason, D. C., Wang, H.-J. and Lohani, B. (2003) Extraction of tidal channel networks from airborne scanning laser altimetry and aerial photography. In: Serpico, S. B. (ed.) Image and Signal Processing for Remote Sensing. SPIE Proceedings, 8 (4885). SPIE Press, Bellingham, WA, pp. 162-169. doi:

https://doi.org/10.1117/12.463200 Available at http://centaur.reading.ac.uk/5889/

It is advisable to refer to the publisher's version if you intend to cite from the work. See Guidance on citing.

Published version at: http://dx.doi.org/10.1117/12.463200

To link to this article DOI: http://dx.doi.org/10.1117/12.463200

Publisher: SPIE Press

All outputs in CentAUR are protected by Intellectual Property Rights law, including copyright law. Copyright and IPR is retained by the creators or other 
copyright holders. Terms and conditions for use of this material are defined in the End User Agreement.

\section{www.reading.ac.uk/centaur}

\section{CentAUR}

Central Archive at the University of Reading

Reading's research outputs online 


\title{
Extraction of tidal channel networks from airborne scanning laser altimetry and aerial photography
}

\author{
David C. Mason ${ }^{\mathrm{a}}$, Hai-Jing Wang ${ }^{\mathrm{a}}$, Bharat Lohani ${ }^{\mathrm{b}}$ \\ ${ }^{a}$ Environmental Systems Science Centre, University of Reading, Reading, UK. \\ ${ }^{\mathrm{b}}$ Department of Civil Engineering, Banaras Hindu University, Varanasi, India.
}

\begin{abstract}
The study of the morphodynamics of tidal channel networks is important because of their role in tidal propagation and the evolution of salt-marshes and tidal flats. Channel dimensions range from tens of metres wide and metres deep near the low water mark to only $20-30 \mathrm{~cm}$ wide and $20 \mathrm{~cm}$ deep for the smallest channels on the marshes. The conventional method of measuring the networks is cumbersome, involving manual digitising of aerial photographs. This paper describes a semi-automatic knowledge-based network extraction method that is being implemented to work using airborne scanning laser altimetry (and later aerial photography). The channels exhibit a width variation of several orders of magnitude, making an approach based on multi-scale line detection difficult. The processing therefore uses multiscale edge detection to detect channel edges, then associates adjacent anti-parallel edges together to form channels using a distance-with-destination transform. Breaks in the networks are repaired by extending channel ends in the direction of their ends to join with nearby channels, using domain knowledge that flow paths should proceed downhill and that any network fragment should be joined to a nearby fragment so as to connect eventually to the open sea.
\end{abstract}

Keywords: digital elevation model, LiDAR, multiscale edge detection, edge association, Ems river, Wrangle Flats

\section{INTRODUCTION}

Tidal channel networks are among the most interesting landforms of coastal embayments and estuaries. These begin from the low water mark and develop through the tidal flats and salt-marshes, dissecting a landscape that is heterogeneous in terms of its different sedimentological and ecological characteristics. To a large extent, these channels control the hydrodynamics of the tidal basin. On ebb tides they drain the marshes and tidal flats, while on flood tides they act as conduits which the incoming water fills prior to flooding the areas around them when the channels are overtopped. The sediment and nutrient exchanges between salt-marshes and tidal flats and between tidal flats and the sea are also controlled through the network of tidal channels.

The study of tidal network morphology assumes significance in view of their role in tidal propagation and the evolution of salt-marshes and tidal flats. The processes governing their development and evolution are currently an open question. Early studies found that their geomorphological characteristics were similar to those of terrestrial river networks, and implied that they were formed by the draining of the tide from the marsh ${ }^{1}$. However, there are fundamental differences between tidal and terrestrial channels, the most important being the bi-directional flow which occurs in tidal channels. Therefore other workers have suggested that tidal channels are not primarily drainage networks, but have developed in response to the sea dissipating tidal and wave energy within the network on the flood tide $^{2}$. Rinaldo et al. ${ }^{3}$ found that parts of the network may be ebb dominated and other parts flood dominated.

For further author information -

D.C.M. (correspondence): email: dcm@mail.nerc-essc.ac.uk ; WWW: http:/www.nerc-essc.ac.uk; Telephone: +44-1189-

318743

H-J.W. email: hjw@mail.nerc-essc.ac.uk

B.L. email: b lohani@sify.com 
Channel dimensions range from several tens of metres wide and several metres deep near the low water mark to only $20-30 \mathrm{~cm}$ wide and $20 \mathrm{~cm}$ deep for the smallest channels on the marshes. The networks may be quite complicated, as their basic tree structures may contain loops and bifurcations and (especially near the low water mark) may exhibit channels within channels.

The conventional method of measuring the networks is cumbersome and subjective, involving digitising aerial photographs in conjunction with field measurement of channel depths and widths for selected parts of the network. Given that typical networks may have a length of over $20 \mathrm{~km} / \mathrm{km}^{2}$, and that several tens of square kilometres may need to be analysed, in general there will be too many channels to be extracted accurately manually. In addition, insufficient information for quantitative measurement of the channels is obtained this way.

Airborne scanning laser altimetry (LiDAR) is an important new data source that can be used for network extraction ${ }^{4}$. A spatial resolution of $0.5 \mathrm{~m}$ is currently possible, coupled with a height accuracy of $15 \mathrm{~cm}$. At this spatial resolution all but the smallest channels will be detected. Use of LiDAR having first and last return data may well allow the smallest channels to be detected, by differencing the first and last return heights within a pixel. The current trend is for LiDAR data to be collected in conjunction with high resolution colour aerial photography or linescanner data, to provide the user with a visual image of the LiDAR DEM. This imagery will detect the smallest channels down to $20 \mathrm{~cm}$.

Aerial photographs of intertidal zones are presently much more commonly available than LiDAR data, and are particularly useful for providing a snapshot of a marsh in the (pre-LiDAR) past for comparison with its present-day state. Although photographs contain no explicit height information, pixels are defined everywhere within the image, whereas with LiDAR no heights are measurable over water due to absorption of the beam.

The LiDAR-derived network morphology should enable geomorphological theories such as that of Allen ${ }^{5}$ to be tested, which proposes that dynamic equilibrium of a salt-marsh should be characterised by a linear increase with seaward distance of total channel cross-sectional area along shore-parallel transects.

This paper describes a semi-automatic network extraction method currently under development, which is being designed to work using either LiDAR or aerial photography alone or with both data sources combined in a fusion approach which should produce an improved channel network.

\section{CHANNEL NETWORK EXTRACTION}

The method is an example of drainage network extraction from a digital elevation model (DEM). There has been extensive research into the development of techniques for extracting river channel networks from low resolution terrestrial DEMs. Examples are the steepest gradient method of Jenson and Domingue ${ }^{6}$ and the multilevel skeletonisation technique of Meisels et al. ${ }^{7}$. The performance of these methods has been shown to be only moderate when applied to LiDAR imagery of tidal channels, due to the different morphological characteristics of fluvial and tidal channels and the very high spatial resolution of $\mathrm{LiDAR}^{4}$.

A multi-level knowledge-based approach is being implemented, whereby low level algorithms first extract channel fragments based mainly on image properties then a high level processing stage improves the network using domain knowledge ${ }^{8}$. The channels exhibit a width variation of several orders of magnitude, making an approach based on multiscale line detection difficult. The approach adopted therefore uses edge detection to detect channel edges, then associates adjacent anti-parallel edges together to form channels. This has the advantage that the low level processing can be made similar for LiDAR and aerial photographs. An alternative approach for LiDAR data would be to determine an adaptive height threshold based on local mean height, then find all below-threshold pixels ${ }^{4}$. This would automatically associate pixels within the channels together, but would not exploit the fact that channels must be linear features. It would also not be applicable to aerial photography, for which a height threshold is meaningless. 


\subsection{Channel network extraction from LiDAR data}

\subsubsection{Low level processing}

\subsubsection{Preprocessing}

Where salt-marshes are present in the inter-tidal zone, the LiDAR returns may contain signals due to short vegetation generally less than $1 \mathrm{~m}$ high. The objective is to search for linear channels in the LiDAR-derived DEM rather than the Digital Surface Model. It may therefore be necessary to remove vegetation heights from the 2-band first- and last-return LiDAR data. Cobby et al. ${ }^{9}$ describe an algorithm using height texture for estimating local ground height and vegetation height from last-return LiDAR data. A suitable $0.5 \mathrm{~m}$ resolution first- and last-return LiDAR image of tidal channels has yet to be acquired for this project. An example 1m-resolution last-return LiDAR image of unvegetated tidal flats on the river Ems, Germany, has been used for system development to date (figure 1). This exhibits a number of tidal channels and shows their width variations, though a crude sea-wall has unfortunately been constructed across the bottom of the area.

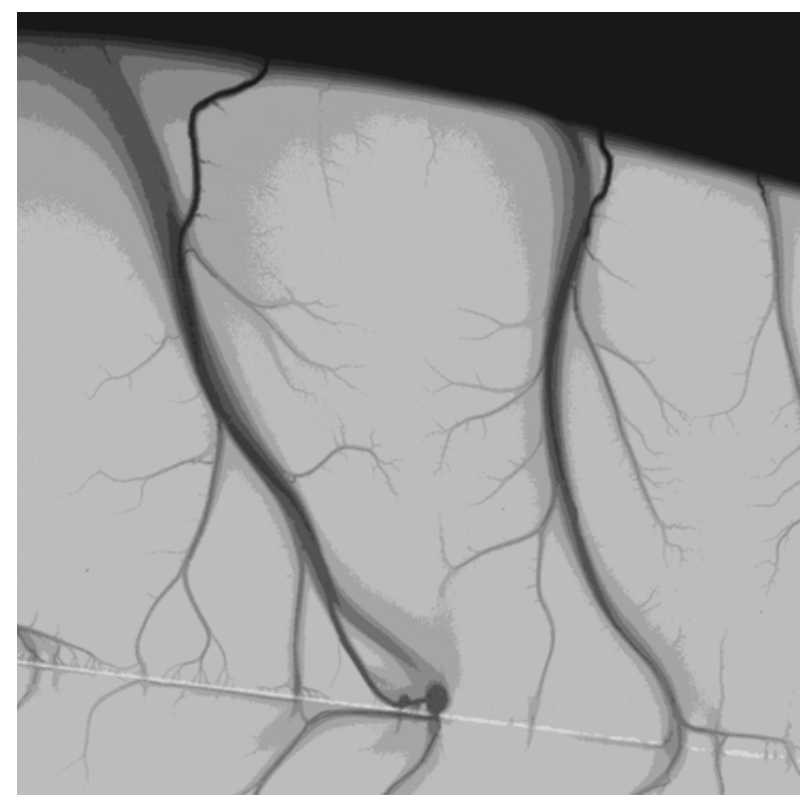

Figure 1. LiDAR height data of channel networks on the river Ems, Germany (lighter = higher)

\subsubsection{Edge detection}

In contrast to the channel width variation of several orders of magnitude, the variation in the slopes at the channel banks spans a narrower range. The majority of the edges of the smaller channels in the salt-marsh and upper tidal flats can be detected using a high frequency 3x3-pixel edge detector, though those of larger channels near the low water mark can only be detected using a low frequency detector. A multi-scale edge detector is therefore used for channel edge detection. This is a modified form of Canny's "feature synthesis" technique using a fine-to-coarse heuristic ${ }^{10}$. The method takes into account the facts that at each image position different width edge operators will give different responses, and that the maximum response from an edge may occur at different places for different operators. It adaptively selects the edge width giving the best trade-off between signal-to-noise ratio and edge localization.

The edges obtained from the small 3x3 Sobel operator are first detected everywhere in the image. These are used provided they are sufficiently strong, since these give the best localization of an edge. The larger operator outputs are synthesised from the Sobel outputs, by taking the edges detected by the small operator in the window, and convolving 
with a Gaussian normal to the central pixel's edge direction, with the standard deviation of the Gaussian being the standard deviation of the larger operator. At each position in the convolution only the component of the edge strength in the direction of the central edge pixel is summed. If the larger operator edge is significantly stronger than the Sobel edge, and the contributions to the larger operator from each side of the central pixel are similar, the larger operator edge strength replaces the Sobel's. Requiring similar contributions to the larger operator output from either side of the central pixel is a means of focusing its response. Most of the low frequency edges are ramp edges, and the similarity constraint ensures that the larger operator response is only selected when it is situated near the centre of the ramp. Canny ${ }^{10}$ applied his method recursively to edges at decreasing scales, but in this case use of only two scales (1 and 8) selects the majority of both high and low frequency channel edges (figure 2a).

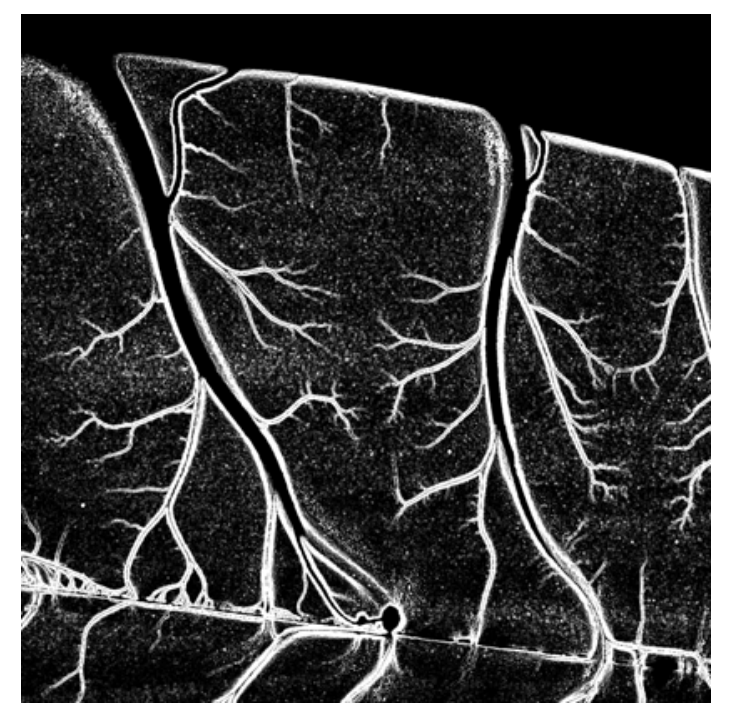

(a)

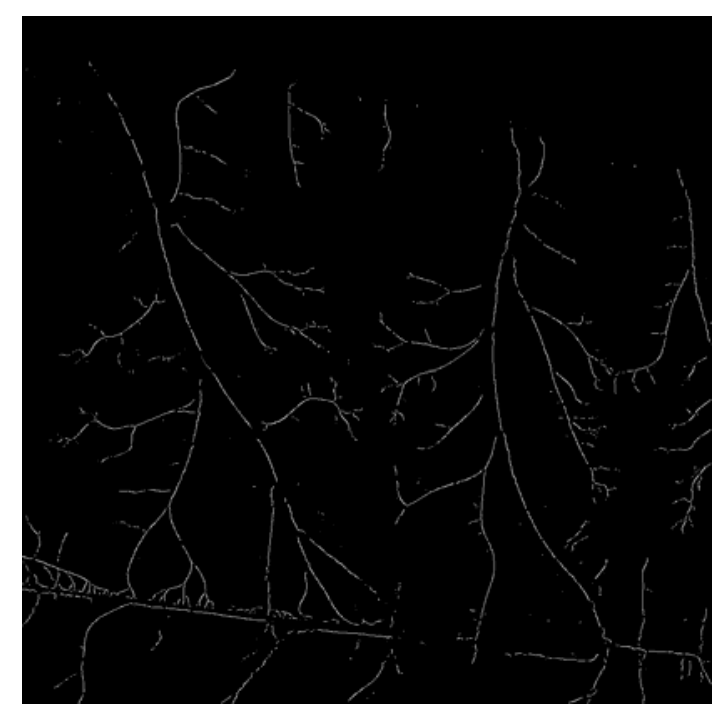

(c)

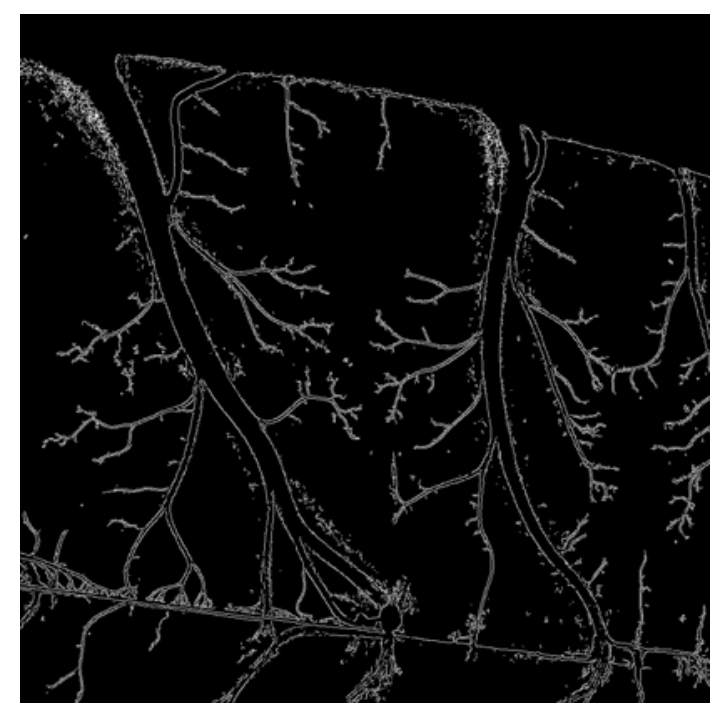

(b)

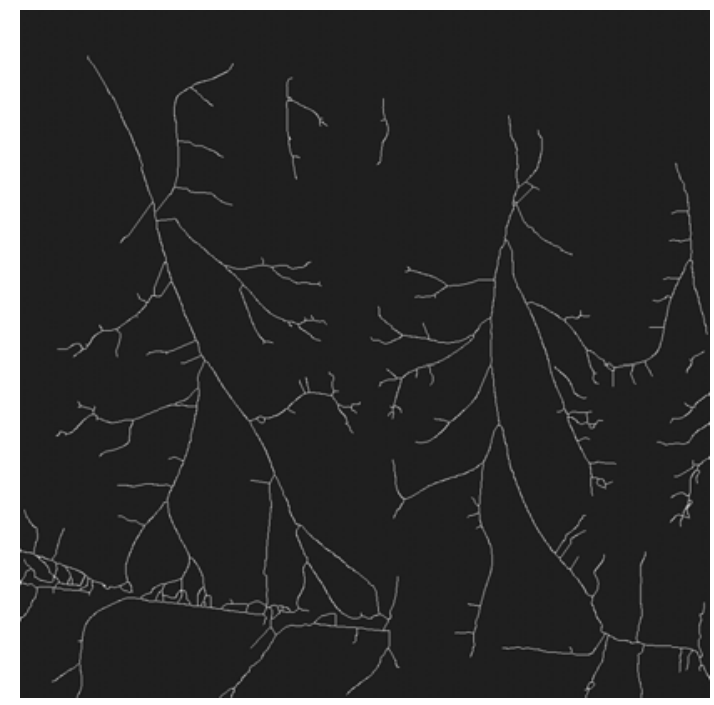

(d)

Figure 2. (a) Combined edges, (b) edges after edge suppression, (c) high score channel centerlines, (d) centerlines after channel repair. 
Edges which are non-maxima along an axis perpendicular to their edge direction are then suppressed. In addition, domain knowledge is introduced to suppress edge maxima lying within the larger channels. For each edge maximum a search is made along a line perpendicular to its edge direction for 25 pixels on either side of the central pixel to see if a significantly higher maximum having the same direction exists. If one is found, and there is no pixel between it and the central pixel having a higher height, the central edge maximum is suppressed. Surviving edge maxima are then thresholded using hysteresis thresholding ${ }^{10}$ and thinned to single pixel width (figure $2 b$ ).

\subsubsection{Edge association}

Edge detection of a tidal channel will generate two anti-parallel edges from either side of it. These must be associated together. Nevatia and $\mathrm{Babu}^{11}$ describe an algorithm to associate vector edges together. Instead, a distance-withdestination transform is used to associate anti-parallel edges together in a raster image. This is a form of distance transform which stores for each pixel its distance to the nearest edge, and also the direction from which the minimum distance was propagated. This allows back-tracking from a pixel to find its nearest edge pixel. The transform is operated upon to identify positions having distances that are maxima or saddle points, by performing non-maximum suppression followed by thinning.

The distance maxima occur at the centrelines of channels, but other maxima of no interest will occur between channels. To differentiate between these two classes of maxima, a score is calculated for each pixel. The overall score is the product of a number of individual scores, which are functions of -

(a) how narrow the edge separation is

(b) how anti-parallel the two adjacent edges are, and whether their slopes both increase moving away from the distance maximum (the latter ensures that, while a channel would score high on this criteria, a raised sea wall would score low)

(c) how low the heights are along the two paths from a maximum to its closest edge points.

A distance maximum's score is big if it is linked with perfectly anti-parallel edges with a narrow separation bounding runs of pixels with heights less than the edge heights, and with heights increasing away from the channel centre. As the channels are always unvegetated whereas their surroundings may be salt-marshes, it would be possible to increase the score if the LiDAR height texture in the channel was low, a characteristic of unvegetated surfaces. Hysteresis thresholding is then used to select long connected strings of pixels having high scores (figure 2c). Note that the raised sea-wall has been removed, while a ditch adjacent to it remains.

\subsubsection{Higher level processing}

At this stage the thresholded score image consists of sets of channel network centrelines with each network consisting of a number of disconnected fragments. Breaks in the networks are repaired by extending channel ends in the direction of their ends to join with nearby channels. Domain knowledge is used at this stage, namely (a) flow paths should proceed downhill (b) any network fragment should be joined to a nearby fragment so as to connect eventually to the open sea.

Connected regions are found in the score image, with small regions being rejected. A confidence is calculated for each region based on the sum of the scores of all pixels making up the region. Each region is a skeleton, and for each skeleton its endpoints are detected. Within its endpoint set, the lowest endpoint is found. This establishes the position of the root of this network fragment's tree. A crude estimate of the height of the waterline (the land-sea boundary) is made by finding the lowest height among the roots of all the fragments.

For each root endpoint significantly above the waterline height, a search for a channel centreline to join with is made by searching forward in a narrow cone whose axis is the direction of the endpoint for a centreline near the endpoint. Candidate centreline pixels must be lower than the root endpoint to be joined with, and have higher scores if they subtend a small angle to the cone axis, have a small separation distance from the endpoint and if both endpoint and candidate regions have high confidences. The candidate having the maximum score above a threshold is selected, and providing one exists is joined to the endpoint. A revised confidence for the modified network is then calculated. 
The repair procedure (figure 2d) acts as a consistency check on the network. If after it any network fragments remain whose root endpoints are significantly above the waterline height, these are not connected to the sea, and are priority candidates for operator interaction, as are networks with low confidences.

After the repair stage, a binary image of the channels is obtained by selecting all pixels between each distance maximum and its associated edges. A preliminary version is shown in figure 3.

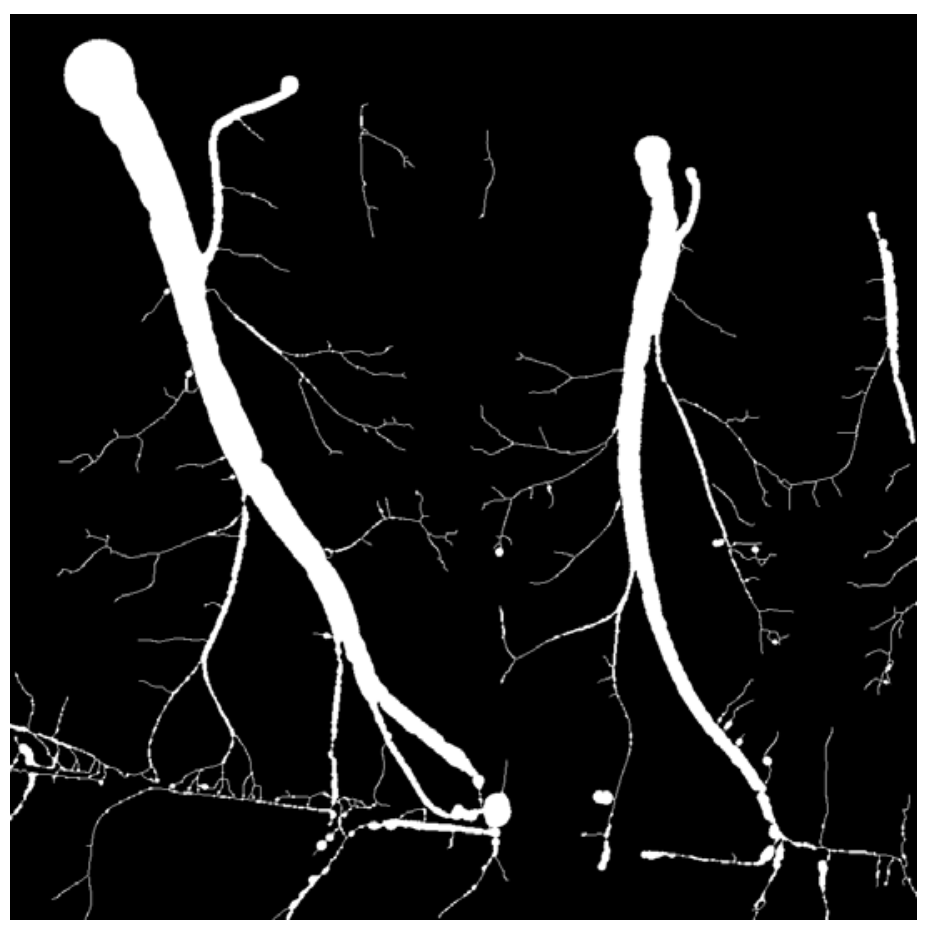

Figure 3. Channels extracted from the LiDAR data.

\subsection{Channel network extraction from aerial photographs}

The technique is currently being extended to extract networks from aerial photographs. These will be limited to colour photographs to make extraction of vegetation and water more straightforward. Nevertheless the task is more difficult than network extraction from LiDAR because aerial photographs are less constrained scenes. Figure 4 shows that the channels may exhibit considerable shading information that could be used in determining presence or absence of channels. Shading effects caused by the varying aspects presented by pixels to the sun are particularly pronounced for channels running perpendicular to the sun azimuth angle. On tidal flats in particular, a DEM could be generated using shape-from-shading techniques ${ }^{12,13}$ if it could be assumed that surface albedo was everywhere constant. If knowledge of sun angle was not available it could be determined from the image ${ }^{14}$. The camera could be assumed to be at nadir. On salt-marshes it would be necessary to screen out vegetated areas prior to performing shape-from-shading in their unvegetated sections. The resulting DEM could then be passed through the previous processing chain to extract networks. In practice, however, surface albedo can vary substantially over tidal flats, partly due to varying sand moisture contents. Instead, experiments with colour edge detectors are currently being performed. The approach being considered is to associate pairs of parallel edges together if they have a common edge. 
All the LiDAR data acquired will have simultaneous air photography or multispectral linescanner associated with it, so it would be short-sighted to develop a channel extraction method only for LiDAR. One advantage of aerial photography is that it should be of higher resolution than the LiDAR data. Another advantage is that it will have areas of water clearly delineated, whereas with the LiDAR undefined areas may be either water or outside the LiDAR coverage. Fusion of edges in the two edge sets detected separately in aerial photographs and LiDAR data is being considered.

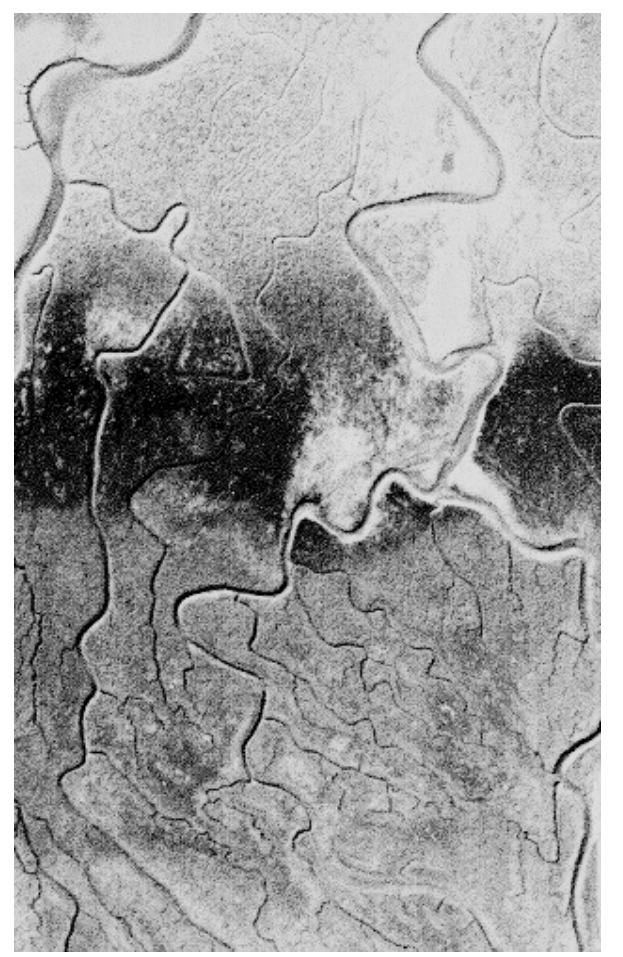

Figure 4. Aerial photograph of channels on Wrangle Flats, The Wash, UK.

\section{CONCLUSION}

A multilevel knowledge-based method of extracting tidal channel networks from LiDAR imagery using multiscale edge detection, edge association and domain knowledge has been developed. The system has been designed to be semiautomatic to allow an operator to correct the errors that will undoubtedly be made. The networks extracted will be used to test geomorphological theories concerning the evolution of tidal flats and salt-marshes.

\section{ACKNOWLEDGEMENTS}

This work is being funded under the EU FP5 TIDE (Tidal Inlet Dynamics and Environment) project and the Italian Government CORILA project. Thanks are due to TopoSys Ltd. for the provision of LiDAR data.

\section{REFERENCES}

1. J.R. French and D.R. Stoddart, "Hydrodynamics of salt-marsh creek systems - implications for marsh morphological development and material exchange”, Earth Surface Processes and Landforms, 17(3), 235-252, 1992.

2. J.S. Pethick, "Saltmarsh geomorphology”, In Saltmarshes, morphology, conservation and engineering significance ((Eds. Allen, J.R.L. and Pye, K). Cambridge University Press, Cambridge, 41-62, 1992. 
3. A. Rinaldo, S. Fagherazzi, S. Lanzoni, M. Marani and W.E. Dietrich, “Tidal networks: 3. Landscape-forming discharges and studies in empirical geomorphic relationships”, Water Resources Research, 35(12), 39005-3917, 1999.

4. B. Lohani and D.C. Mason, "Application of airborne scanning laser altimetry to the study of tidal channel geomorphology”, ISPRS J. Photogrammetry \& Remote Sensing, 56, 100-120, 2001.

5. J.R.L. Allen, "Morphodynamics of Holocene salt marshes: a review sketch from the Atlantic and southern North Sea coasts of Europe”, Quaternary Science Reviews, 19, 1155-1231, 2000.

6. S.K. Jenson and J.O. Domingue, "Extracting topographic structure from digital elevation data for geographic information system analysis”, Photogrammetric Engineering and Remote Sensing 54(11), 1593-1600, 1988.

7. A. Meisels, S. Raizman and K. Arnon, “Skeletonizing a DEM into a drainage network”, Computers and Geosciences 21(1), 187-196, 1995.

8. F. Wang and R. Newkirk, “Knowledge-based system for highway network extraction”, IEEE Trans. Geoscience and Rem. Sens., 26(5), 525- 530, 1988.

9. D.M. Cobby, D.C. Mason and I.J. Davenport, "Image processing of airborne scanning laser altimetry data for improved river flood modelling”, ISPRS J. Photogrammetry \& Remote Sensing, 56, 121-138, 2001.

10. J.F. Canny, “A computational approach to edge detection”, IEEE Trans. PAMI, 8(6), 679-698, 1986.

11. R. Nevatia and KR. Babu, "Linear feature extraction and description”, Computer Graphics and Image Processing, 13, 257-269, 1980.

12. B.K.P. Horn and M.J. Brooks, Shape from shading. MIT Press, Cambridge, MA, USA. 1989.

13. C. Ichoku, A. Karnielli, A. Meisels and J. Chorowicz, "Detection of drainage channel networks on digital satellite images”, Int. J. Rem. Sens. 17(9), 1659-1678, 1996.

14. M.J. Brooks and B.K.P. Horn, “Shape and source from shading”, Proc. IJCAI, Los Angeles, CA, 932-936, August 1985. 